\title{
A clinicopathological study of atypical leiomyomas: Benign variant leiomyoma or smooth-muscle tumor of uncertain malignant potential
}

\author{
IOANNIS KALOGIANNIDIS ${ }^{1}$, THOMAS STAVRAKIS ${ }^{1}$, THEMISTOKLIS DAGKLIS ${ }^{1}$, STAMATIOS PETOUSIS $^{1}$, \\ CHRISTINA NIKOLAIDOU ${ }^{2}$, IOANNIS VENIZELOS ${ }^{2}$ and DAVID ROUSSO ${ }^{1}$ \\ ${ }^{1}$ Third Department of Obstetrics and Gynecology, Aristotle University of Thessaloniki; \\ ${ }^{2}$ Department of Pathology, Hippokration General Hospital, Thessaloniki 54642, Greece
}

Received October 4, 2014; Accepted June 25, 2015

DOI: $10.3892 / \mathrm{ol} .2015 .4062$

\begin{abstract}
Atypical leiomyomas are histopathologically recognized by moderate to severe pleomorphic atypical tumor cells showing low mitotic counts without coagulative tumor cell necrosis. The histopathological features and clinical behavior of these tumors are unclear. The surgical management of these lesions includes myomectomy and hysterectomy. The current study presents 5 cases of women with atypical leiomyomas that were managed surgically. The clinicopathological characteristics of the patients and recurrences were analyzed. The median age of the patients was 43 years old. While 3 out of 5 patients were treated by hysterectomy (with or without salpingo-oophorectomy), the remaining 2 patients were treated by myomectomy. The median mitotic index was 3 mitotic figures/10 high-power fields. The post-operative course of all patients was uneventful, and there was no evidence of local or distant recurrence in a median follow-up time of 72 months. The treatment of choice for atypical leiomyomas is hysterectomy. However, myomectomy may be an option for fertility sparing cases. A thorough consultation with regard to the uncertain behavior of such tumors and a rigorous follow-up must be offered in such patients.
\end{abstract}

\section{Introduction}

Leiomyomas (fibroids) are the most frequent benign masses of the female genital tract, occurring in almost $25 \%$ of the general female population (1). Uterine leiomyosarcomas (LMS), on the

Correspondence to: Dr Thomas Stavrakis, Third Department of Obstetrics and Gynecology, Aristotle University of Thessaloniki, 49 Konstantinoupoleos, Thessaloniki 54642, Greece

E-mail: tstavrakis@gmail.com

Key words: smooth-muscle tumor of uncertain malignant potential, uterine leiomyomas, atypical leiomyomas, leiomyosarcomas other hand, represent $\sim 8 \%$ of all uterine malignancies (2). The pre-operative detection of LMS is difficult due to the clinical similarity with ordinary leiomyomas.

The classification of uterine smooth-muscle tumors is based on the assessment of three histopathological characteristics: Mitotic count activity [mitotic figures per 10 high-power fields (MFs/10 HPFs)], presence or absence of coagulative tumor cell necrosis, and degree of cytological atypia (2). Any uterine smooth-muscle tumor with an unusual combination of the aforementioned histopathological features that does not satisfy the diagnostic crit eria of leiomyomas or LMS according to the 2003 World Health Organization (WHO) classification of tumors of the female genital organs (mesenchymal tumors and associated lesions) is defined as a uterine smooth-muscle tumor of uncertain malignant potential (STUMP) (3). The atypical leiomyomas are a special category of smooth-muscle tumors that are considered to be STUMPs by certain studies, while others consider them to be separate histopathological entity of smooth-muscle tumors for which there is limited experience or a low risk of recurrence $(4,5)$.

The aim of the present case series was to add to the understanding of these tumor classifications using the 10-year experience of a single institution in the management of atypical leiomyomas.

\section{Materials and methods}

Data collection. The clinical and histopathological records of the Third Department of Obstetrics and Gynecology, Aristotle University of Thessaloniki (Thessaloniki, Greece) were reviewed for the period between 2002 and 2012. Data were collected carefully, and only patients with a histopathological diagnosis of an atypical leiomyoma, independently of the surgical approach (myomectomy or hysterectomy with/ without adnexectomy), were included in the present analysis. The Institutional Ethics Committee of Aristotle University of Thessaloniki approved this research and the Institutional Review Board approved the present study.

Tumor definition. The histopathological criteria that were used for the definition of atypical leiomyomas were based on the 
Table I. Summary of clinical histological findings of atypical leiomyomas of the study.

\begin{tabular}{lccccccccc}
\hline Case & $\begin{array}{c}\text { Patient } \\
\text { age, years }\end{array}$ & $\begin{array}{c}\text { No. of } \\
\text { tumors }\end{array}$ & $\begin{array}{c}\text { Tumor } \\
\text { size, cm }\end{array}$ & MI & \multicolumn{1}{c}{ Atypia } & $\begin{array}{c}\text { Tumor cell } \\
\text { necrosis }\end{array}$ & $\begin{array}{c}\text { Type of } \\
\text { surgery }\end{array}$ & $\begin{array}{c}\text { Follow-up, } \\
\text { months }\end{array}$ & Status \\
\hline 1 & 46 & 4 & 6.0 & 4 & Focal moderate to severe & No & Hysterectomy & 132 & AWND \\
2 & 43 & 1 & 5.0 & 2 & Focal moderate to severe & No & Hysterectomy & 108 & AWND \\
3 & 42 & 1 & 4.0 & 3 & Focal moderate to severe & No & Hysterectomy & 72 & AWND \\
4 & 43 & 1 & 4.0 & 5 & Multifocal moderate to severe & No & Myomectomy & 28 & AWND \\
5 & 26 & 1 & 6.5 & 3 & Diffuse moderate to severe & No & Myomectomy & 15 & AWND \\
\hline
\end{tabular}

MI, mitotic index; AWND, alive with no disease.

2003 WHO classification (3) as follows: Focal (or multifocal) or diffuse moderate to severe nuclear atypia, an absence of coagulative tumor cell necrosis and a mitotic index (MI) of 0-10 MFs/10 HPFs.

Patient characteristics. The characteristics of patient age at the time of treatment, the main clinical sign or symptom at the time of diagnosis, tumor size (maximum diameter) and the number of uterine tumors were reviewed. Furthermore, histopathological characteristics such as cellular atypia, tumor cell necrosis and MI were also assessed.

One of the main scopes of this study was also to record the clinical outcome of the patients, particularly in cases with recurrent disease.

\section{Results}

Clinical findings. In total, 5 patients with atypical leiomyomas were identified during the period of the study. The median age of these patients was 43 years (range, 26-46 years) at the time of surgery. While 3 of the patients (cases 1-3) complained of heavy menstrual bleeding (menorrhagia), a uterine tumor was identified during a routine gynecological ultrasound in the remaining 2 patients (cases 4 and 5). In one case (case 1), a hysterectomy confirmed the presence of multiple leiomyomas, as previously suspected from the ultrasound examination. All clinical findings are presented in Table I.

One of the patients (case 1) underwent a total abdominal hysterectomy with a bilateral salpingo-oophorectomy (BSO), while a total hysterectomy without BSO was the treatment of choice for cases 2 and 3 . A myomectomy was performed in cases 4 and 5, via laparoscopy and laparotomy, respectively. The surgical approach in the cases of myomectomy (laparoscopy vs. laparotomy) was exclusively based upon the surgeon's decision. A conservative approach was opted for in case 4 , as the preservation of fertility was desired, and in case 5 , due to the patient's young age and in order to preserve the any childbearing ability. These two patients underwent comprehensive counseling with regard to the risk of recurrence following a conservative approach and informed consent was routinely obtained from all patients prior to the surgical approach.

Pathological findings. In all 5 cases, the tumors were labeled as atypical leiomyomas based on their histopathological

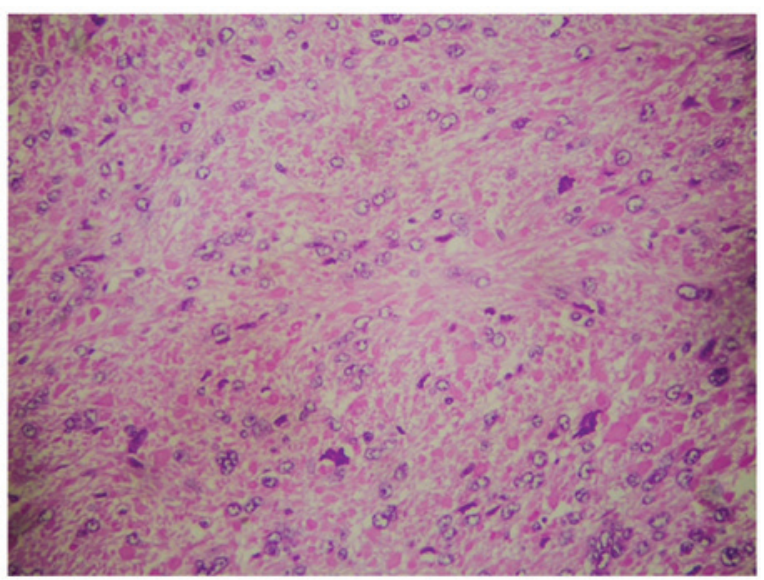

Figure 1. Case 5: Focus of the tumor with the cells showing moderate to severe atypia. Mitoses are $\sim 3 / 10$ high-power field. There are no sites of coagulative necrosis (hematoxylin and eosin stain; magnification, x200).

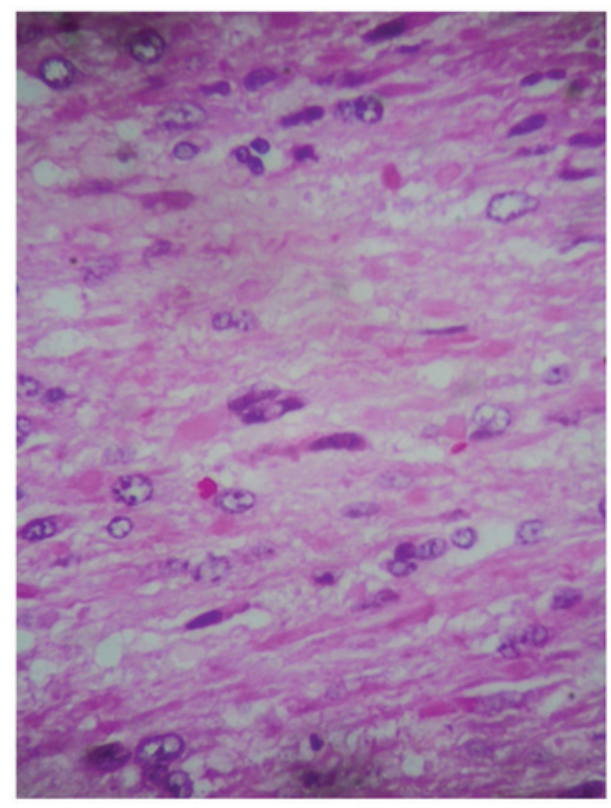

Figure 2. Case 5: Focus of the smooth muscle neoplasm, where the tumor cells exhibit moderate to severe atypia (hematoxylin and eosin stain; magnification, $\mathrm{x} 400)$.

characteristics (Figs. 1 and 2). In the case with multiple leiomyomas (case 1), 1 leiomyoma was found to be atypical 
and the remaining 3 were characterized as ordinary leiomyomas. The median diameter of the tumors was $4.5 \mathrm{~cm}$ (range, 4-6 cm) and the median MI was $3 \mathrm{MF} / 10 \mathrm{HPFs}$ (range, 2-5 MF/10 HPFs).

Clinical outcomes. Clinical follow-up was available for all patients for a median period of 72 months (range, 15-132 months). The post-operative course of all patients was uneventful and no recurrences were observed during the follow-up period.

\section{Discussion}

Atypical leiomyomas are uterine smooth-muscle tumors of controversial nature for clinicians and pathologists. It is unclear whether they represent a completely benign variant of typical leiomyomas or uterine STUMPs.

The term STUMP, according to the WHO definition for tumors of the uterine corpus, includes the smooth-muscle tumors that do not fulfill the histopathological criteria for typical leiomyomas or LMSs, actually being neither benign nor malignant (3). The histopathological criteria required for the definition of atypical leiomyomas are the absence of coagulative tumor cell necrosis, a MI of $<10 \mathrm{MFs} / 10 \mathrm{HPFs}$ and diffuse, focal or multifocal moderate to severe atypia. Clearly there is an overlapping of the definition between the two entities.

Bell et al (Stamford study), in a historical study of 213 patients with problematic uterine smooth-muscle neoplasms, did not use the term 'STUMP' (4). However, according to the diagnostic strategy used, four groups of problematic uterine smooth-muscle tumors were distinguished with unclear clinical behavior. More specifically, cases with diffuse moderate to severe cellular atypia, without coagulative cell necrosis and an MI of $<10 \mathrm{MFs} / 10 \mathrm{HPFs}$ were found and termed 'atypical leiomyomas with low risk of recurrence', while cases with focal or multifocal moderate to severe atypia, without cell necrosis and an MI of $<20 \mathrm{MFs} / 10$ HPFs were characterized as 'atypical leiomyomas with limited experience'. Furthermore, cases without atypia or with mild atypia, with no coagulative cell necrosis and an MI of $\geq 20 \mathrm{MFs} / 10 \mathrm{HPFs}$ were defined as 'leiomyomas with increased MI but with limited experience', and tumors without atypia or with mild atypia, with an MI of $<10 \mathrm{MFs} / 10 \mathrm{HPFs}$ and with coagulative cell necrosis were defined as 'smooth-muscle tumors with low malignant potential' (4).

However, cases similar to the latter histopathological entities were characterized have been characterized as STUMPs by other studies. For example, Fletcher (6) defined uterine tumors with diffuse or multifocal moderate to severe cytological atypia with an MI of $<10$ but $>7$ as STUMPs, while Bell et al (4) defined them as atypical leiomyomas with a low risk of recurrence. The literature by D'Angelo and Prat (7), and Nucci and Oliva (8) is also in agreement with Nucci's terminology. In addition, Zaloudek (9) defined a STUMP as a uterine smooth-muscle tumor without cell necrosis, an MI of $\geq 5$ and focal moderate to severe cytological atypia, a definition that overlaps with the 'atypical leiomyomas of limited experience' in the Stanford study (4).
Another crucial controversy relates to the patients with 'leiomyomas with bizarre nuclei'. These are a specific histopathological category with an extremely low MI of $<2 \mathrm{MFs} / 10 \mathrm{HPFs}$, and showing similarities with atypical leiomyomas (coagulative necrosis and cytological atypia) with regard to other histopathological characteristics. Certain studies have suggested that leiomyomas with bizarre nuclei are an almost benign variant of typical leiomyomas (10), while the WHO classification includes leiomyomas with bizarre nuclei in the definition of atypical leiomyomas (3).

It is clear that there is a wide range of histopathological characteristics of atypical leiomyomas. The key question is whether it is meaningful to classify uterine smooth-muscle tumors with extremely similar characteristics in different groups with different terminology. In our opinion, this is indeed crucial for the prognosis of the patient. The recurrence rate in the group of patients with 'leiomyoma with bizarre nuclei' is non-existent according to the literature. In an analysis of 24 patients with leiomyomas with bizarre nuclei treated by hysterectomy $(n=20)$ and myomectomy $(n=4)$, Downes and Hart (10) demonstrated no evidence of recurrence after a mean follow-up time of 11.2 years. The MI in this study ranged from 0 to 2.8 (mean, 0.8). Similar results were found by Evans et al (11). By contrast, smooth-muscle tumors with a MI range of 0 to $10 \mathrm{MFs} / 10 \mathrm{HPFs}$, without coagulative cell necrosis and diffuse or focal/multifocal significant (moderate to severe) atypia, actually 'atypical leiomyomas', showed different clinical outcomes with regard to the prognosis of the patients. In Stanford's study, from the 46 patients with a diagnosis of an 'atypical leiomyoma with a low risk of recurrence' (no coagulative necrosis with diffuse significant atypia and a variable MI of 0-10 MFs/ $0 \mathrm{HPFs}$ ) who underwent a hysterectomy or myomectomy, one of the patients developed extensive pelvic and abdominal relapse two years after a hysterectomy. The patient was further treated with debulking and adjuvant chemotherapy and radiotherapy, and she was alive with disease 60 months after the second treatment. All the other patients were alive in a follow-up time ranging from 24 to 116 months (4). However, in the same study another three cases initially treated conservatively with myomectomy underwent a subsequent hysterectomy due to a residual tumor, which was identical with the tumor that was recognized in the myomectomy specimens (4). No recurrent disease was noted after hysterectomy in the former patients. The present analysis in a similar group of patients demonstrated no recurrences in a median follow-up time of 72 months (range, 15-132 months). In another previous study, 1 out of 13 patients with atypical leiomyomas, all treated by myomectomy, presented with local recurrence at the site of primary myomectomy 22 months after the initial treatment. The patient was again managed by myomectomy and remained alive without evidence of disease at 35 months after the second myomectomy. However, no relapse was recorded for any other patients during the follow-up period (5). In the present study, no relapse was observed in the two patients who were treated conservatively in a median follow-up time of 22 months.

Laparoscopic myomectomy was performed in one case from the present study. Laparoscopic myomectomy appears to be more advantageous compared with laparotomic myomectomy, 
as it is associated with reduced post-operative pain, shorter hospitalization and a lower risk of post-operative adhesions $(12,13)$. However, there is a lack of evidence concerning the relapse rate of patients with atypical leiomyomas treated by myomectomy via laparoscopy.

The age of the patient is extremely important in the investigation of uterine smooth-muscle tumors. Women diagnosed with LMSs are on average almost 10 years older than those with leiomyomas, and the majority of them are $>40$ years old (9). The age of women diagnosed with STUMP and a subsequent recurrence is lower than the age of women characterized by an uneventful follow-up. Ip et al showed a difference of 3 years between the patient group of STUMPs with subsequent recurrence (mean age, 44.5 years) compared with those without recurrence (mean age, 47.5 years) (14). Similarly, in another study, the patients without relapse were on average 10 years older (mean age, 43.9 years) compared with those with recurrence (mean age, 33.7 years) (15). Atypical leiomyomas are rare in postmenopausal women (9). The mean age in the present study group was 43 years old, which is in accordance with the previous studies.

The majority of leiomyomas are asymptomatic and diagnosed during routine gynecological examinations. Submucosal leiomyomas are the most frequent cause of abnormal bleeding (menorrhagy). Large intramural leiomyomas may be associated with infertility or chronic pelvic pain (16). The palpation of leiomyomas is difficult unless their diameter is large $(16,17)$. Imaging techniques, such as computed tomography or magnetic resonance imaging, are not able to distinguish whether the mass is benign or malignant (18). Therefore, to date, there is no reliable method to pre-operatively clarify the specific category into which a uterine smooth-muscle tumor belongs.

The present study is not without limitations, the most important being the small number of patients and the short follow-up time, particularly for the patients treated conservatively.

In conclusion, when a histopathological diagnosis of atypical leiomyoma follows a myomectomy, a hysterectomy should be the treatment of choice. When fertility preservation is important, a myomectomy may remain a treatment option, although a comprehensive consultation with the patient, concerning the risk of recurrent disease and the close-up that will be required, must be undertaken. Further studies with longer follow-up periods and a larger number of samples are required in order to confirm or reject the present results associated with the safety of myomectomy for patients with atypical leiomyoma.

\section{References}

1. Echt G, Jepson J, Steel J, Langholz B, Luxton G, Hernandez W, Astrahan $\mathrm{M}$ and Petrovich Z: Treatment of uterine sarcomas. Cancer 66: 35-39, 1990.
2. Kho KA and Nezhat $\mathrm{CH}$ : Evaluating the risks of electric uterine morcellation. JAMA 311: 905-906, 2014.

3. Hendrickson MR, Tavassoli FA,Kempson RL, et al: Mesenchymal tumours and related lesions. In: World Health Organization Classification of Tumours: Pathology and Genetics of Tumours of the Breast and Female Genital Organs. Tavassoli FA and Devilee P (eds). IARC Press, Lyon, pp236-243, 2003.

4. Bell SW, Kempson RL and Hendrickson MR: Problematic uterine smooth muscle neoplasms A clinicopathologic study of 213 cases. Am J Surg Patholo 18: 535-558, 1994.

5. Sung CO, Ahn G, Song SY, Choi YL and Bae DS: Atypical leiomyomas of the uterus with long-term follow-up after myomectomy with immunohistochemical analysis for p16INK4A, p53, Ki-67, estrogen receptors and progesterone receptors. Int J Gynecol Pathol 28: 529-534, 2009.

6. Fletcher CD: Tumors of female genital tract: Myometrium. In: Diagnostic Histopathology of Tumors. Nucci M (ed). 3rd edition. Churchill Livingstone Elsevier, Philadelphia, pp690-692, 2003.

7. D'Angelo E and Prat J: Uterine sarcomas: A review. Gynecol Oncol 116: 131-139, 2010.

8. Nucci MR and Oliva E: Pure mesenchymal and mixed Mullerian tumors of the uterus. In: Gynecologic Pathology. Oliva E (ed). 1st edition. Churchill Livingstone Elsevier, Philadelphia, p289, 2009.

9. Zaloudek CJ, Hendrickson MR and Soslow RA: Mesenchymal tumors of the uterus. In: Blaustein's Pathology of the Female Genital Tract. Kurman RJ, Ellenson LH and Ronnett BM (eds). 6th edition. Springer, New York, pp453-527, 2011.

10. Downes KA and Hart WR: Bizarre leiomyomas of the uterus: A comprehensive pathologic study of 24 cases with long-term follow-up. Am J Surg Pathol 21: 1261-1270, 1997.

11. Evans HL, Chawla SP, Simpson C and Finn KP: Smooth muscle neoplasms of the uterus other than ordinary leiomyoma. A study of 46 cases, with emphasis on diagnostic criteria and prognostic factors. Cancer 62: 2239-2247, 1988.

12. Bulletti C, Polli V, Negrini V, Giacomucci E and Flamigni C: Adhesion formation after laparoscopic myomectomy. J Am Assoc Gynecol Laparosc 3: 533-536, 1996.

13. Dubuisson JB, Fauconnier A, Chapron C, Kreiker G and Nörgaard C: Second look after laparoscopic myomectomy. Human Reprod 13: 2102-2106, 1998.

14. Ip PP, Cheung AN and Clement PB: Uterine smooth muscle tumors of uncertain malignant potential (STUMP): A clinicopathologic analysis of 16 cases. Am J Surg Pathol 33: 992-1005, 2009.

15. Guntupalli SR, Ramirez PT, Anderson ML, Milam MR, Bodurka DC and Malpica A: Uterine smooth muscle tumor of uncertain malignant potential: A retrospective analysis. Gynecol Oncol 113: 324-326, 2009

16. Buttram VC Jr and Reiter RC: Uterine leiomyomata: Etiology, symptomatology and management. Fertil Steril 36: 433-445, 1981.

17. Lumsden MA and Wallace EM: Clinical presentation of uterine fibroids. Bailliere Clin Obstet Gynaecol 12: 177-195, 1998.

18. Sato K, Yuasa N, Fujita M and Fukushima Y: Clinical application of diffusion-weighted imaging for preoperative differentiation between uterine leiomyoma and leiomyosarcoma. Am J Obstet Gynecol 210: 368, e1-8, 2014. 